\title{
From homicide inquiries to high secure hospital inquiries: a decade of social and political restrictive pressure on forensic psychiatry
}

\author{
CERI EVANS and DEJI OYEBODE
}

The dominant theme for forensic psychiatry in the UK that emerged from the 1990's from clinical and research perspectives was one of unmet needs for patients in all 5 main settings for the specialty (high security; medium security; low security/local forensic services; prison; court diversion schemes). The dominant theme from a legal, political and social perspective, over the same time period, was a strongly increasing insistence on significant lowering of the risk presented by forensic patient groups to the public. Forensic psychiatry now finds itself struggling to find clinical resources for those patients it does want to treat and manage but can't; and struggling to resist political initiatives for it to manage those patients in ways it does not want to. Our specialty, therefore, is under pressure. In this situation, it is sensible to derive perspective and reference points from an overview. Our intention here is to briefly lay out some of the unmet clinical needs and service provision deficits which have been identified for our patients over the last decade or so, to follow this with a selective summary of the legal changes and Inquiry body findings over a similar time scale, and to then suggest that an ethical framework is the crucial overview for forensic psychiatry to consider in its currently state.

The High Secure Hospitals have been the focus for increasingly heated and polarised debate over the last 10 years. Amid significant organisational changes in which the then Special Hospital Service Authority (SHSA), followed by 'independently managed provider units' (in association with the 'purchaser' High Security Psychiatric Services Commissioning Board (HSPSCB), became responsible for managing the 3 High Secure Hospitals, two clinical themes with organisational implications stand out. First, a move from a milieu with

Indirizzo per la corrispondenza: Dr. D. Oyebode, Forensic Mental Health Services, Shaftesbury Clinic Medium Secure Unit, London SW17 7DJ (United Kingdom).

E-mail: oyebode@swlstg-tr.nhs.uk custodial overtones (partly attributable to majority staff membership with the Prison Officers Association (POA) (Murphy, 1997)), to one with clinical emphasis was required, and partially achieved, by the changes to management structures for these hospitals (Higgins, 1996). Second, various estimates identified major 'overcontainment' of patients within the high secure settings, such that, at least from a security perspective, between one-third and one-half of all patients did not require this level of containment (Special Hospitals Services Authority, 1995; Maden et al., 1995; Baxter, 1996; Bartlett et al., 1996).

The difficulties in establishing a welfare attitude by staff and in achieving appropriate transfer to lower levels of security for a significant proportion of patients, has implied the need for much closer integration of the High Secure hospitals with regional NHS facilities (e.g. Baxter, 1996; Exworthy, 1998), .conceivably allowing for facilitated vertical transfer and reduction of geographic and clinical isolation. Some have even argued for the closure of the High Secure Hospitals altogether (Bluglass, 1992). Others have highlighted the demand for high-secure beds, the inability of medium levels of security to contain significant numbers of patients, treatment-resistant patients, serious re-offenders (all negative reasons), and the asylum function i.e. catering for minority groups and diversity of meaningful daytime activity (positive reasons), as indications for the continuation of the High Secure Hospitals (Tidmarsh, 1998). The option of replacing the Special Hospitals with more numerous, geographically distributed, NHSintegrated units has been championed e.g. Bluglass (1992), although this approach has been criticised as unrealistic given the formidable expense, the predicted repetition of the difficulties facing the established institutions, and lack of empirical support for this alternative (Tidmarsh, 1998; Gunn, 1999). The controversy surrounding the High Secure Hospitals has been accentuated by recent Inquiries (see later).

Medium Secure Unit (MSU) resources are currently inadequate to cope with the demand for beds, 
particularly long-term placements. The Butler Committee (Home Office and Department of Health and Social Security, 1975) was instrumental in initiating the regional medium secure unit programme by combining clarity and brevity in expressing the need for such units, with forceful reasoning for the need for a central funding mechanism (Grounds, 1996). However, the story of the 1990's has been one of both uneven and inadequate access to these units, and longer duration of admissions than was originally intended to be the case. Research on the pattern of admissions to MSU's has suggested that there is a bias towards mentally ill remand prisoners being admitted for assessment and treatment at the exclusion of other groups, including personality disordered remand prisoners and High Secure Hospital patients of all legal categories (Murray, 1996). A clear service gap has been identified in the provision of longterm medium secure beds amounting to well over 1000 long-term secure placements (Taylor et al., 1996; Reed, 1997), which is correlated with a significant proportion, perhaps up to $70 \%$ of patients, staying in these facilities for longer than the 2-year period initially targeted (Mohan et al., 1997; Murray 1996).

In comparison to the MSU programme instigated at the recommendation of the Butler Committee, no equivalent guidance and urgency has been articulated for local forensic services. Grounds (1996) has been forthright in criticism of the Reed Committee (Department of Health and the Home Office, 1992), because it 'should have recognised that now the substantial gap is in local provision. This is the main location of unmet needs and absent services'. He saw the key step following elucidation of the need, as the pressure for government to provide targeted funding for defined (e.g. intensive community support, assertive outreach, out-of-hours services and crisis intervention, and rapid assessment services for courts and the police) and costed models of a district-based forensic service (as opposed to the regional-based medium secure units), because the Butler Committee had recognised that local health authorities could not be relied upon to financially support these kind of services, given competing demands (ibidem). There is little research data to inform the discussion of the level of need in this area; the need is apparent although unspecified, but the obstacles are challenging for both purchaser and provider for forensic patients within the community. Providers are challenged by transgression of normal admission criteria by patients with offending histories, substance misuse, personality difficulties, and question marks over treatability. Health purchasers are challenged by the lack of clear statements of specific needs, and acceptable indicators of effectiveness of clinical interventions for this group, particularly as criminal justice and social objectives crossover with clinical needs (ibidem).

In a dynamic reminiscent of the difficulties facing the High Secure Hospitals (and implying an emphasis of 'custodial' over 'care' by the POA), the UK prison services have struggled to balance their security and treatment roles to attain a therapeutic environment for mentally disordered offenders. That prison medical officers may have irresolvable inherent ethical conflict between their legal duties as doctors and as prison officers was recognised early (Bowden, 1976; Eastman, 1993), although the Prison Inspectorate was still addressing this issue in recent years, controversially arguing that the NHS should provide medical services for prisoners (HM Inspectorate of Prisons for England and Wales, 1996). What has been clearly established is that epidemiological studies of prison populations consistently reveal high levels of psychiatric morbidity and unmet need. For instance, an interview survey of 5\% of sentenced male prisoners found $2 \%$ to be psychotic and $3 \%$ to be in need of NHS transfer (Gunn et al., 1991). Comparative point prevalences of psychiatric disorder for the remanded male prison population found $5 \%$ to be psychotic and $9 \%$ to be in need of NHS transfer (Brooke et al., 1996). The Office for National Statistics (ONS) recently reported that $7 \%$ of women and $14 \%$ of men in the convicted prison population, and $10 \%$ of both men and women in the remanded population, had suffered from a psychotic episode in the previous 12 months (Office for National Statistics, $1998 \mathrm{a}, \mathrm{b}$ ). If the declared standard that the mentally ill in prison should receive parity in quality of care with comparable NHS patients is to be accepted, there are obviously large gains to be made in current service provision in prisons before this standard is met.

The Court Diversion Services were introduced 10 years ago in line with the policy of 'diversion and discontinuance' (Department of Health and Home Office, 1990). The magistrate's court location of these services was chosen because they were near the 'beginning' of the criminal justice process, and were positioned to provide a cost-effective filter through which all cases must pass (Joseph, 1992). There are now about 150 such schemes operating in England and Wales, with a further 40 schemes being based in police stations. Although there are notable exceptions (e.g. James \& Hamilton, 1991), a recent review concluded that most Court Diversion Schemes were inadequately planned, organised or resourced, and, therefore, they 
were having only limited effect (James, 1999). It was acknowledged that different aspects of their functioning needed to be evaluated to get a full picture, but barely 1 in 10 schemes operated effectively in their primary purpose of diverting mentally disordered offenders into hospital beds. This was linked to the fact that most schemes did not have admitting rights or access to secure beds, with the result being that they were not having much impact on the numbers being admitted from the criminal justice system. The most successful schemes were senior psychiatrist-led with access to beds, but most schemes were community psychiatric nurse (CPN) or trainee psychiatrist led (ibidem). James (1999) judged that although the Reed report and Circular 66/90 had increased awareness of the issues surrounding diversion, no central coordination was in place, which had contributed to poorly designed local initiatives. He concluded that the 'experimental' phase of court diversion schemes in the 1990's should now end, with the introduction of central strategy involving integration with core local psychiatric supervision, use of senior staff, and development of clear operational policies and audit monitoring processes being necessary for the actual survival of most schemes (ibidem).

Against this foreground of unmet need with deficits and defects in service provision, it is revealing to examine the legal backdrop which helps to define forensic psychiatry (Eastman, 1993); it reveals a series of legislative enactments in the last decade or so, which are individually restrictive, and collectively protectionist. The Supervision Register (HMSO, 1994), introduced in April 1994, was criticised by the then President of the Royal College of Psychiatrists on a range of fronts, including: it was a politically motivated response to public disquiet over much publicised tragedies; it was unlikely to have significant effect on clinical practice, it was expected to be costly; it would be time consuming to administer; it would have an adverse effect on the therapeutic relationship; and it would provide a disincentive (perceived potential scapegoating) to become a key worker (Caldicott, 1994). This criticism was not isolated (e.g. Holloway, 1994), and there is evidence that negative feelings within the profession about the Supervision Register remain, even in view of possibly more assertive outreach by key workers for 'a small group of vulnerable patients', as encouraged by the formal documentation process (Vaughn, 1998).

The Mental Health (Patients in the community) Act $1995(x)$ had as its principle provision a 'Supervised Discharge Order (SDO)' which aimed to ensure that certain patients discharged from compulsory detention received appropriate aftercare, by requiring a patient to live at a specified address, allow access to the supervisor and other professionals, and to attend for specified treatment (allowing a 'power to convey' capacity but not compulsory treatment capacity for the treating team). There was also a robust negative response to this Act e.g. by the Registrar of the Royal College of Psychiatrists, who called it «...the worst of both worlds. The mentally ill will be subject to the power of 'arrest' and to no apparent purpose. Psychiatrists remain deeply sceptical...» (Thompson, 1995)», which became legally effective on 1 April 1996. Serious concern was raised that the emphasis was wrong, and that «legal rules are unlikely to compensate for inadequate community resources or professional skill and training (Eastman. 1997)».

The Crime (Sentences) Act 1997, with the associated «Hospital and Limitation Direction», has, similarly, been widely criticised by the psychiatric community, in this case as a distortion of the original «hybrid order" envisaged by the Reed Committee (e.g. Reed, 1994; Eastman, 1996). Indeed, Eastman (ibidem) went so far as to call the Home Office's proposal (at that stage) 'the single most important proposal to confront British forensic psychiatry since the Percy Commission (1957)...' The original intention of the Reed Committee was to provide a cautious strategy for the transfer of those patients belonging to the legal category psychopathic disorder who were of uncertain treatability, so that clinicians would not find themselves attempting to indefinitely manage psychopaths who proved to be untreatable. However, as the law was eventually enacted this emphasis was lost, and the legal rationale for introducing a hybrid order was focused on legal culpability and public safety instead of uncertain treatability. In addition, the hybrid orders are potentially applicable to those suffering from other forms of mental disorder e.g. mental illness. The concern is now that there has been a substantial shift in balance from welfare to a justice agenda, and that civil rights of patients might be affected (e.g. through indefinite detention without recourse to a Mental Health Review Tribunal). Ultimately, the reluctance of mentally disordered offenders to place themselves in a position where they are vulnerable to being made subject to a hybrid order may even expose the current deficiency of appropriate psychiatric defences for charges less than murder (Eastman \& Peay, 1998).

If the legal framework in which forensic psychiatry was operating represented an inexorable development of 
a protectionist approach to policy and legislation (sometimes at the expense of civil rights), the plethora of Inquiries into 'homicides in the community' which characterised particularly the first half of the 1990's had an unmistakeable effect in favouring defensive practise by, and producing demoralisation in, forensic psychiatrists. In 1994, the Department of Health issued a circular making it mandatory to have an inquiry if the perpetrator of homicide had a history of recent psychiatric or social care (Department of Health, 1994). This particular method of examining tragedies of this kind has been the subject of much intense criticism. Although some feel that the levels of homicide risk are high enough to warrant a concerted approach (Tidmarsh. 1997), others are of the view that the risk is insignificant compared to more common causes of death e.g. suicide, road traffic accidents (Szmukler, 2000), and Section 2 manslaughter may even be falling (Taylor \& Gunn, 1999).

There is real concern that only limited lessons can be learnt from a series of single case studies which are based on rare events (homicide), particularly when the process is vulnerable to dubious causal attribution and hindsight bias (Bowden, 1995, Szmukler, 2000). Moreover, although there are strong reasons for continuing with statutory inquiries e.g. helping the bereaved families, there is little doubt that there are wide ranging costs, which weigh heavily on the patient, the family and the psychiatric services (Szmukler, 2000). There are now strong calls for a super-ordinate approach to the inquiries ('an audit of Inquiries: Enquiry, not Inquiries', Eastman, 1996) and more systematic research aimed at evaluating potential deficiencies cited by previous Inquiries across a range of services (Petch \& Bradley, 1997). The National Confidential Inquiry into Homicides and Suicides by People with a Mental Illness (Appleby et al., 1999), a Department of Health funded Royal College of Psychiatry undertaking, has partially addressed these calls, and this willingness of the Royal College to investigate its own 'failures' may have placed it in a unique position internationally, in terms of the seriousness with which it is treating these issues (Thompson, 1999). However, even though the alleged relationship between homicide by mentally disordered offenders and community care has been deemed spurious (Szmukler et al., 1999), and reservations exist about the apparent UK assertion that such homicides are preventable (e.g. Smukler's (1999) memorable comment, that '...colleagues from other countries show surprise and ask why. They know that preventing homicides is like stopping accidents and wars. We do our best to try to reduce their likelihood, but no one has ever (our italics) stopped them. They are part of the human condition'), the statutory Inquiries have continued, although they are scheduled to stop.

Alongside the homicide Inquiries, there have also been contentious Special Hospital inquiries, the most recent of which has contributed to the current heated debate concerning Dangerous and Severe Personality Disorder (DSPD) (Fallon et al., 1999). The Fallon report (ibidem) into the Hospital's Personality Disorder Unit, concluded that the root cause of many of Ashworth Hospital's problems was actually related to the implementation of some of the earlier Blom-Cooper et al's (1992) Inquiry recommendations for liberalisation concerning the management of the mentally ill at the same institution. Following complaints from absconded patient, the report described serious and unacceptable breakdown in security, the circulation of pornography, the use of alcohol and illicit drugs, the operation of 'businesses' by inpatients, and the potential grooming of a visiting female child for abuse. Maden (1999) was scathing in his review of the report, for what he saw as unwarranted and unsustainable extensions of its remit, to review personality disorder and its treatment, and the reform of high secure services. Maden was specifically critical of the recommendation to close the whole hospital (the Inquiry concerned the Personality Disorder Unit), and the inclusion on the panel of a recently retired forensic psychiatrist who had previously declared views that High Secure Hospitals should be closed (ibidem). Gunn (1999) went so far as to say that the report resembled a 'witch hunt' in its treatment of professionals, because it made no attempt to mitigate those mistakes made by staff.

The leading issue currently facing forensic psychiatry in the UK is how to manage people with serious personality disorders, which, at a sweep, captures the tensions outlined above between clinical concerns about service provision, facilities and knowledge, sociopolitical concerns about public safety, and the ongoing climate of 'failure' review. The clinicians are concerned about the lack of knowledge about effective methods of assessment and treatment of personality disordered offenders (Cope et al., 1993; Dolan \& Coid, 1993). The politicians are concerned to (be seen to) lower risk to the public and have created a new label (DSPD) in association with policy aimed at detaining people in this non-scientific category who may be both untreatable and unconvicted, in new specialist institutions which would be hybrids of prison and hospital (Home Office and Department of Health, 1999; Straw, 1998; Kendall, 
1998). Ultimately, these patients would be detained under mental health criteria to satisfy the 'unsound mind' requirement of the European Convention of Human Rights (article 5), for detaining unconvicted people (Eastman, 1999). Although there is broad agreement between the Royal College of Psychiatrists and much of the Government's proposals, there is fierce opposition to its transparent preventive detention aspects (Chiswick, 1999, Eastman, 1999; Mullen, 1999).

In searching for some sense of perspective in criticising the government's proposals, clinicians have referred to ethical principles in making their respective cases (e.g. Maden, 1999a,b; Eastman, 1999; Mullen, 1999). The questions that are being asked of our specialty drive to the core of what we do; our roles, responsibilities based on ethical values, our relationship with other agencies. At the moment, the strong sense is that the majority of forensic psychiatrists are uncomfortable with the government's proposals regarding DSPD, at least in their entirety. Under this sort of pressure, super-ordinate reference points encourage clarity of thinking, and the ethical framework seems right in this regard.

This kind of ethical discourse has occurred before within forensic psychiatry, and a review of the debate around the true dilemmas involved in the preventive detention of patients with violent fantasies but no violent behaviour, which took place about two decades ago, has obvious relevance to the current debate. The debate was concerned with the question: is it ever morally justifiable to detain an unconvicted individual just because he is dangerous and might commit a violent act in the future? The debate was essentially between two opposing philosophical camps, which have been labelled 'the protectionists' and the 'libertarians'. The protectionists asserted that it is justifiable on the grounds of public protection to detain a dangerous individual, even if he or she is unconvicted. A major proponent of this position laid out the possible reasons for detention (retribution, social disapproval, deterrence, treatment, and public protection), and at the time of writing concluded that, given the limited success of treatment and the tendency for courts to impose relatively short sentences (akin to the present day situation whereby courts impose only $2 \%$ of all possible life sentences), the only possible justification for long-term detention was for public protection (Walker, 1982). He developed his case for endorsement of this principle as acceptable by arguing that it is morally wrong to detain individuals in these circumstances only if it is morally wrong to make 'false' positive mistakes. The analogy given was that it would be hard to call it morally wrong to remove a potentially cancerous growth only to find it was benign: in the same way, being wrong about the prediction of dangerousness should be acceptable to the public as a point of principle.

Walker (1982) (Hamilton \& Freeman, 1982) also pointed out a second fallacy, equating a 'false negative' with a 'false positive'. In terms of dangerousness, his position would be that a false positive (detention of an individual who wouldn't have been violent) is not as bad as a false negative (releasing an individual who was thought not to be dangerous, but who did then commit a violent act). He goes on to argue from a 'non-absolutist' position, that if an unconvicted person was to be detained on the basis of dangerousness, then the judgement should not be based on an isolated piece of behaviour (a pattern of behaviour would be required), it would be important to inspect alternative methods of managing the risk e.g. supervision orders, and it would be essential to provide tolerable conditions of detention.

The libertarian position, in contrast, asserts that it is not morally justifiable to have unconvicted but dangerous individuals long-term incarcerated on the basis of dangerousness. This position was elaborated by Bottoms (1982), who cited three main reasons for the established over prediction of dangerousness by clinicians: the pressure to 'err on the side of caution'; loss of the crucial perspective that it is not individuals who are dangerous, but, rather, individuals in certain situations (which might only arise rarely) who behave dangerously; and the base-rate phenomenon (the very low rate of violent events per se leading to an overestimation of 'dangerous' judgements. He then couches the dilemma as the detention of false positives versus the 'missing' of false positives, and uses the hypothetical situation: ' 1 man out of 3 will be dangerous, but we don't know which one: should we lock them all up?' to argue that we should not.

Bottom's (1982) starting position was to suggest that 'justice' has a position of primacy in social institutions, along the lines that 'truth' does in the field of academia and knowledge. If an individual could show that their position was supported by the principle of justice, then it would take a very strong justification to undermine that position. It is, he argued, 'just', that an individual should not be detained if he is unconvicted. He said that the principle of justice should also apply to convicted individuals in that the individual should not be detained any longer than justification by retribution (as opposed to public protection). He stated that there was, therefore, a prima facie case that an injustice has been done to a detained unconvicted individual, which must be 
overcome, but in reality, any challenge is likely to be undermined by our poor accuracy of predictions, for the reasons outlined above.

The level of clinical difficulty in assessing and managing seriously personality disordered individuals who sometimes act violently, and the gravity of the associated ethical dilemmas, may have been underestimated by the current government in the rush for public protection. This debate reflects a decade of increasing legal and political constraints and restrictive pressure on the practice of forensic psychiatry. It is useful to be reminded that the moral integrity of our clinical judgement can be reduced by the social pressures of the day (Pearson, 1999). Currently, the socio-political agenda is public protection, as evidenced by the government's electorate-correct response. The reality is that it is highly debatable and contentious whether or not it is ethical to detain someone for an act they might commit.

Our specialty, therefore, really is under pressure. The necessary clinical resources to achieve the transparent objectives have not matched the restrictive imperative from outside the profession. Moreover, there are strong reservations that this is what we should be doing anyway, although this should be counterbalanced by the perspective that forensic psychiatry as a profession has been slow to understand, let alone embrace, the very nature of our inter-disciplinary position, particularly the legal interface (Eastman, 1993). That this position might just be ethically untenable, or, at least, irresolvable, was described a considerable time ago (Bowden, 1976). The tension which has now been generated, which is underpinned by this inherent ethical dilemma, may be responsible for what has been described as forensic psychiatry's greatest challenge: 'recruitment, recruitment, and recruitment' (Gunn, 1999).

\section{REFERENCES}

Appleby L., Shaw J., Amos T., McDonnell R., Davis S., Harris C., McCann K., Kiernan K., Bickley H. \& Parsons R. (1999). Safer Services: Report of the National Confidential Inquiry into Suicide and Homicide by People with a Mental Illness. Department of Health: London.

Bartlett A., Cohen A., Backhouse A., Highet N. \& Eastman N. (1996). Security needs of SW Thames Special Hospital patients: 1992 and 1993. No way out? Journal of Forensic Psychiatn 7. 256-270.

Baxter R. (1996). Changes in the funding and organisation of the Special Hospitals: should they be 'trust'ed? Journal of Forensic Psychiatry 7, 156-160.

Blom-Cooper L. (1992). Report of the Committee of Inquiry into Complaints about Ashworth Hospital. Cm 2028. HMSO: London.
Bluglass R. (1992). The Special Hospitals (should be closed). British Medical Journal 305, 323-324.

Bottoms A.E. (1982). Selected issues in the dangerousness debate In Dangerousness: Psychiatric Assessment and Management (ed. J.R. Hamilton and H. Freeman). Gaskell: London.

Bowden P. (1976). Medical practice: defendants and prisoners. Journal of Medical Ethics 24, 163-172.

Bowden P. (1995). Confidential inquiry into homicides and suicides by mentally ill people: a preliminary report on homicide. Psychiatric Bulletin 19, 65-66.

Brooke D. Taylor C., Gunn J. \& Maden A. (1996). Point prevalence of mental disorder in unconvicted male prisoners in England \& Wales. British Medical Journal 313, 1524-1527.

Caldicott F. (1994). Supervision registers: the College's response. Psychiatric Bulletin 18, 385-388.

Chiswick D. (1999). Preventive detention exhumed-and enhanced. Psychiatric Bulletin 23, 703-704.

Cope R. (1993). A survey of forensic psychiatrists views on psychopathic disorder. Journal of Forensic Psychiatry 4, 215-236.

Department of Health (1994). Guidance on the Discharge of Mentally Disordered People and their Continuing Care in the Community. Department of Health: London.

Department of Health and the Home Office (1990). Provision for Mentally Disordered Offenders. Circular 66/90. Home Office: London.

Department of Health and the Home Office (1992). Review of Health and Social Services for Mentally Disordered Offenders and Others requiring Similar Services. Final summary Report (the Reed Report). Cm 2088. HMSO: London.

Department of Health and the Home Office (1999). Managing Dangerous People with Severe Personality Disorder. Home Office: London.

Dolan B. \& Coid J. (1993). Psychopathic and Antisocial Personality Disorders: Treatment and Research Issues. Gaskell: London.

Eastman N (1993). Forensic psychiatric services in Britain: a current review. International Joumal of Law and Psychiatry 16, 1-26.

Eastman N. (1996). Hybrid orders: an analysis of their likely effects on sentencing practice and on psychiatric practice and services. Joumal of Forensic Psychiatry 7, 481-494.

Eastman N. (1997). The Mental Health Act (Patients in the Community) Act 1995, a clinical analysis. British Journal of Psychiatry 170, 492-496.

Eastman N. (1999). Public health psychiatry or crime prevention? British Medical Journal 318, 549-551.

Eastman N. \& Peay J. (1998). Sentencing psychopaths: is the «Hospital and Limitation Direction» an ill-considered hybrid? Criminal Law Review 93-108

Exworthy T. (1998). Institutions and services in forensic psychiatry. Journal of Forensic Psychiatry 9, 395-412.

Fallon P., Bluglass R., Edwards B. \& Daniels G. (1999). Report of the Committee of Inquiry into the Personality Disorder Unit, Ashworth Special Hospital. Cm 4195. HMSO: London.

Grounds A. (1996). Forensic psychiatry for the millennium. Journal of Forensic Psychiatry 7; 221-227.

Gunn J. (1999). Ashworth Revisited. British Medical Journal 318, 271.

Gunn J., Maden A. \& Swinton M. (1991). Mentally Disordered Offenders. Home Office: London.

Hamilton J.R. \& Freeman H. (1982). Dangerousness: Psychiatric Assessment and Management. Gaskell: London.

Higgins J. (1996). Future of the special hospitals. Criminal Behaviour and Mental Health 6, Suppl, 65-72. 
HM Inspectorate of Prisons for England and Wales (1996). Patient or Prisoner? A New Strategy for Health Care in Prison. Home Office: London.

HMSO (1994). Introduction of Supervision Registers for Mentally III People. HSG (94) 5: London.

Holloway F (1994). Supervision registers: recent government policy and legislation. Psychiatric Bulletin 18, 593-596.

Home Office and Department of Health (1999). Managing Dangerous People with Severe Personality Disorder. Home Office \& Department of Health: London.

Home Office and the Department of Health and Social Security (1975). Report of the Committee on Mentally Abnormal Offenders (the Butler Report). Cmd 6244. HMSO: London.

James D. (1999). Court Diversion at 10 years: can it work, does it work and has it a future? Joumal of Forensic Psychiatry 10(3), 507-524.

James D.V. \& Hamilton L.W. (1991). The Clerkenwell Scheme: assessing efficacy and cost of a psychiatric liaison service to a magistrate's court. British Medical Journal 303, 282-285.

Joseph PL (1992) Psychiatric assessment at the Magistrates Court. Home Office: London.

Kendall R. (1998). Jack Straw and police under fire after Stone verdict. Times 29 October, 21.

Kershaw C. \& Renshaw G. (1998). Statistics of mentally disordered offenders, England \& Wales 1997. Home Office Statistical Bulletin 19.

Maden A. (1999a). The Ashworth Inquiry: the lessons for psychiatry. Psychiatric Bulletin 23: 455-457.

Maden A. (1999b). Treating offenders with personality disorder. Psychiatric Bulletin 23, 707-710.

Maden T., Curle C., Meux C., Burrow S. \& Gunn J. (1995). The Treatment and Security Needs of Special Hospital Patients. Whurr: London.

Mohan D., Murray K., Taylor P. \& Streed P. (1997). Developments in the use of Regional Secure Unit Bedsover a 12-year period. Journal of Forensic Psychiatry 8, 321-335.

Mullen P. (1999). Dangerous people with severe personality disorder. British Medical Journal 319, 1146-1147.

Murphy E. (1997). The future of Britain's High Security Hospitals. British Medical Journal 314, 1292-1293.

Murray K. (1996). The use of beds in NHS Medium Secure Units in England. Journal of Forensic Psychiatry 7, 504-524.

Office for National Statistics (1998a). Psychiatric Morbidity among Prisoners in England \& Wales. ONS: London.
Office for National Statistics (1998b). Social Trends 28. ONS: London. Pearson G. (1999). Madness and moral panics. In Law without Enforcement: Integrating Mental Health and Justice (ed. N. Eastman and J. Peay). Hart Publications: Oxford.

Percy Commisssion (1957). Report of the Royal Commission on the Law Relating to Mental Illness and Mental Deficiency 1954-57. Cmd 169. HMSO: London.

Petch E. \& Bradley C. (1997). Learning the lessons from homicide inquiries: adding insult to injury? Journal of Forensic Psychiatry 8 , 161-184.

Reed J. (1994). Report of the Department of Health and Home Office Working Party on Psychopathic Disorder Department of Health? Home Office: London.

Reed J. (1997). The need for longer-term psychiatric care in medium or low security. Criminal Behaviour and Mental Health 7. 201-212.

Special Hospitals Service Authority (1995). Service Strategies for Secure Care. SHSA: London.

Straw J. (1998). Straws riposte on mental treatment. Times 31 October, 21.

Szmukler G. (2000). Homicide inquiries-what sense do they make? Psychiatric Bulletin 24, 6-10.

Szmukler G., Thornicroft G., Holloway F. \& Bowden P. (1999). Homicides and community care: the evidence. British Journal of Psychiatry 174, 564-565.

Taylor P.J. \& Gunn J. (1999). Homicides by people with mental illness: myth and reality British. Journal of Psychiatry 174, 9-14.

Taylor P.J., Maden A. \& Jones D. (1996). Long-term medium security hospital units: a service gap of the 1990's. Criminal Behaviour and Mental Health 6, 213-229.

Thompson C. (1995). New powers for care of mentally ill. Times, 23 March.

Thompson C. (1999). The Confidential Inquiry comes of age. British Journal of Psychiatry 175: 301-302.

Tidmarsh D. (1997). Psychiatric risk, safety cultures and homicide inquiries. Journal of Forensic Psychiatry 8, 138-151.

Tidmarsh D. (1998). Asylums or crude cauldrons of containment? The future of the Special Hospitals. Journal of Forensic Psychiatry 9. 505-508.

Vaughn P.J. (1998). Supervision register in practice. Psychiatric Bulletin 22; 412-415.

Walker N. (1982). Ethical aspects of detaining dangerous people. In Dangerousness: Psychiatric Assessment and Management (ed. J.R. Hamilton and H. Freeman). Gaskell: London. 